\title{
AN ANOMALOUS VARIATION DUE TO B CHROMOSOMES IN RYE
}

\author{
R. NEIL JONES* and H. REES \\ Department of Agricultural Botony, University College of Woles, Aberystwyth
}

Received 10.vi.68

\section{INTRODUCTION}

$B$ CHRomosomes in rye are heterochromatic in that they replicate their DNA later in interphase than do the $A$ chromosomes (Darlington and Haque, 1966). In common with other heterochromatic chromosomes the $B$ 's carry no genes of major effect. They are not, however, inert. They cause a delay in germination (Moss, 1966) and in flowering (Kishikawa, 1965), a general reduction in plant vigour and in fertility (Müntzing, 1963) and, at cell level, influence the distribution of chiasmata at meiosis (Jones and Rees, 1967) and the composition and organisation of interphase nuclei in somatic tissue (Jones and Rees, 1968).

While, in general, the influence of $B$ chromosomes upon the rye phenotype is proportional to the number of $B$ 's carried, the following evidence indicates that their genetic activity is not explainable in terms of straightforward additive effects. The indication is that the information vested in $B$ 's is expressed differentially according to whether they are present in odd or even numbers in the nucleus.

\section{The evidence}

(a) Cytological

\section{(i) Chiasma distribution at meiosis}

It has been shown that the variation in chiasma frequency (of $A$ chromosomes) between pollen mother cells (p.m.c.'s) increases with increasing $B$ frequency (Jones and Rees, 1967). This effect is demonstrated in fig. 1, where the chiasma frequency variation between p.m.c.'s within plants is expressed as a variance. Figure 1 also shows that the variances increase in an up-and-down, zig-zag fashion with increasing numbers of $B$ 's. Variances are disproportionately high in the presence of odd as compared with even numbers. A simple test shows that the probability of obtaining such a consistent zig-zag pattern is very low. Thus, it will be seen that the variances for 0 and $1 B$ plants are low and high respectively. The probability of the next class being low is a $\frac{1}{2}$, the next high $\left(\frac{1}{2}\right)^{2}$, and so on. To maintain the high-low sequence for all subsequent $B$ classes the probability is $\left(\frac{1}{2}\right)^{7}$, viz. $0 \cdot 008$, less than one in a hundred.

A slightly more elaborate test confirms the difference in variances between odd and even numbered $B$ 's. In fig. 2 the data have been transformed to logs. On this scale the relationship between variance and $B$ class approaches linearity and consequently permits analysis by linear regression.

* Present address: Sub-Department of Genetics, The Queen's University, Belfast. R 2 
Three regression lines appear in the figure. The first (i) takes into account variances from all $B$ classes. The second (ii) applies to odd numbered $B$ plants, the third (iii) to even numbered $B$ plants. All three regressions are significant and do not differ significantly in respect of slope. What is, however, of particular interest is a comparison of the means of the regressions for odd and even numbers. The comparison is slightly complicated because the odd and even classes along the $\mathrm{X}$ axis are mutually exclusive. A co-variance analysis (table 1) takes account of this. As will be seen the difference between the mean variances is significant $(P=<0.05)$. This

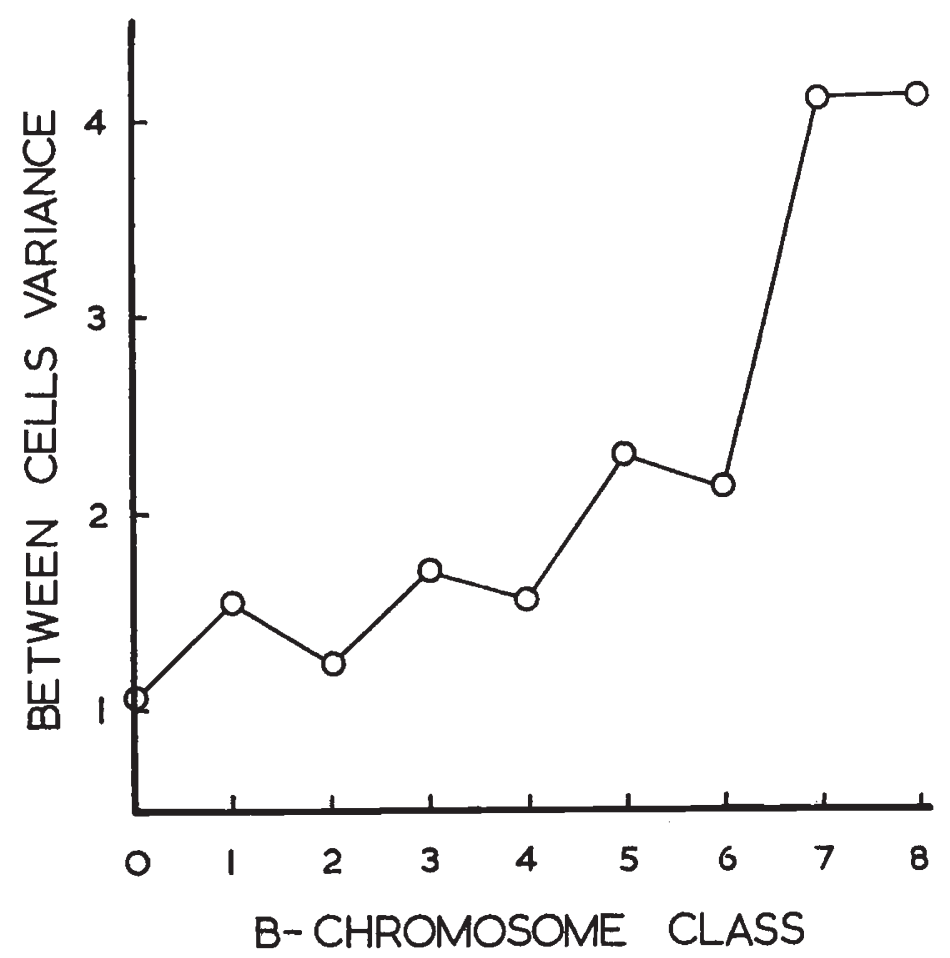

Frg. 1.-Mean " cell variances" within plants plotted against the number of $B$ 's per plant.

confirms the earlier conclusion of a differential influence of $B$ 's upon chromosome behaviour at meiosis according to whether they are present in odd or even numbers.

\section{(ii) The composition of somatic interphase nuclei}

With the addition of $B$ chromosomes to the normal rye complement the nuclear DNA content, as would be expected, increases in direct proportion to the number of $B$ 's added (Jones and Rees, 1968). Nuclear material other than DNA does not, however, increase in strictly direct proportion to $B$ frequency (see Jones and Rees, loc. cit.). This is made conveniently clear in fig. 3 where the ratio of nuclear DNA amount to the total nuclear dry mass (TDM) is plotted against the changing $B$ frequency. The rigidly alternating, zig-zag pattern for odds and evens is manifestly obvious and a 
co-variance analysis of the kind described in the previous section confirms a significant difference between the means of the regressions of $\frac{\mathrm{DNA}}{\mathrm{TDM}}$ on to odd and even $B$ frequencies ( $\mathrm{P}=<0 \cdot 05)$.

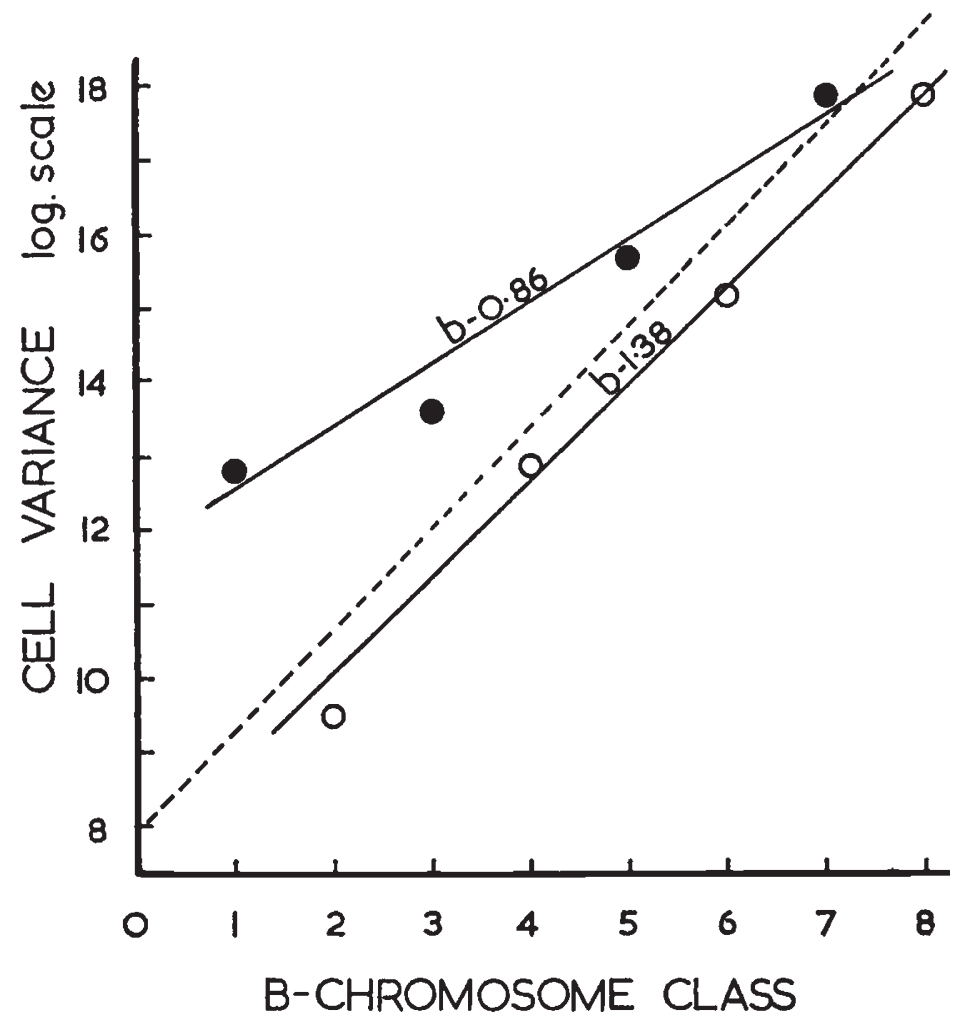

Fig. 2.-Mean " cell variances" (transformed to logs) plotted against: (i) all $B$-chromosome classes, -..--; (ii) odd-numbered $B$-classes only, $B$-classes only, $\mathrm{O}-\mathrm{O}$.

\section{TABLE 1}

Analysis of co-variance of the difference in mean between the regression lines for between cells $\log \log$ variance plotted against odd and even numbered B-chromosome classes respectively

\begin{tabular}{|c|c|c|c|c|c|c|c|c|c|}
\hline Item & $S S x$ & $S P x y$ & SSy & $\begin{array}{l}\text { Regression } \\
\text { SS. }\end{array}$ & $\begin{array}{c}\text { Residual } \\
\text { SS. }\end{array}$ & d.f. & Variance & V.R. & P. \\
\hline 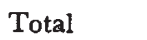 & $42 \cdot 0$ & 42.520 & $56 \cdot 186$ & 43.046 & $13 \cdot 140$ & 6 & - & - & - \\
\hline & $2 \cdot 0$ & $-2 \cdot 28$ & $2 \cdot 61$ & - & 9.763 & 1 & 9.763 & 14.46 & $<0.05$ \\
\hline ror & $40 \cdot 0$ & 44.805 & 53.575 & $50 \cdot 198$ & $3 \cdot 377$ & 5 & 0.675 & - & - \\
\hline
\end{tabular}

The implications of the changes in nuclear dry mass due to $B$ 's have been discussed at length in the paper referred to above. For the present the only point we wish to emphasise is the differential influence of $B$ 's in odd and even combinations.

To find out whether the pattern of variation revealed by our own cytological investigations is of real significance, rather than a bizarre coincidence, we have carried out a survey of other results which deal with 
the influence of $B$ chromosomes on the expression of more familiar, morphological aspects of the rye phenotype.

\section{(b) Morphological}

In figs. 4 and 5 are summarised some of the data of Müntzing (1963) on the effects of $B$ 's upon variation in plant weight, straw height and tiller

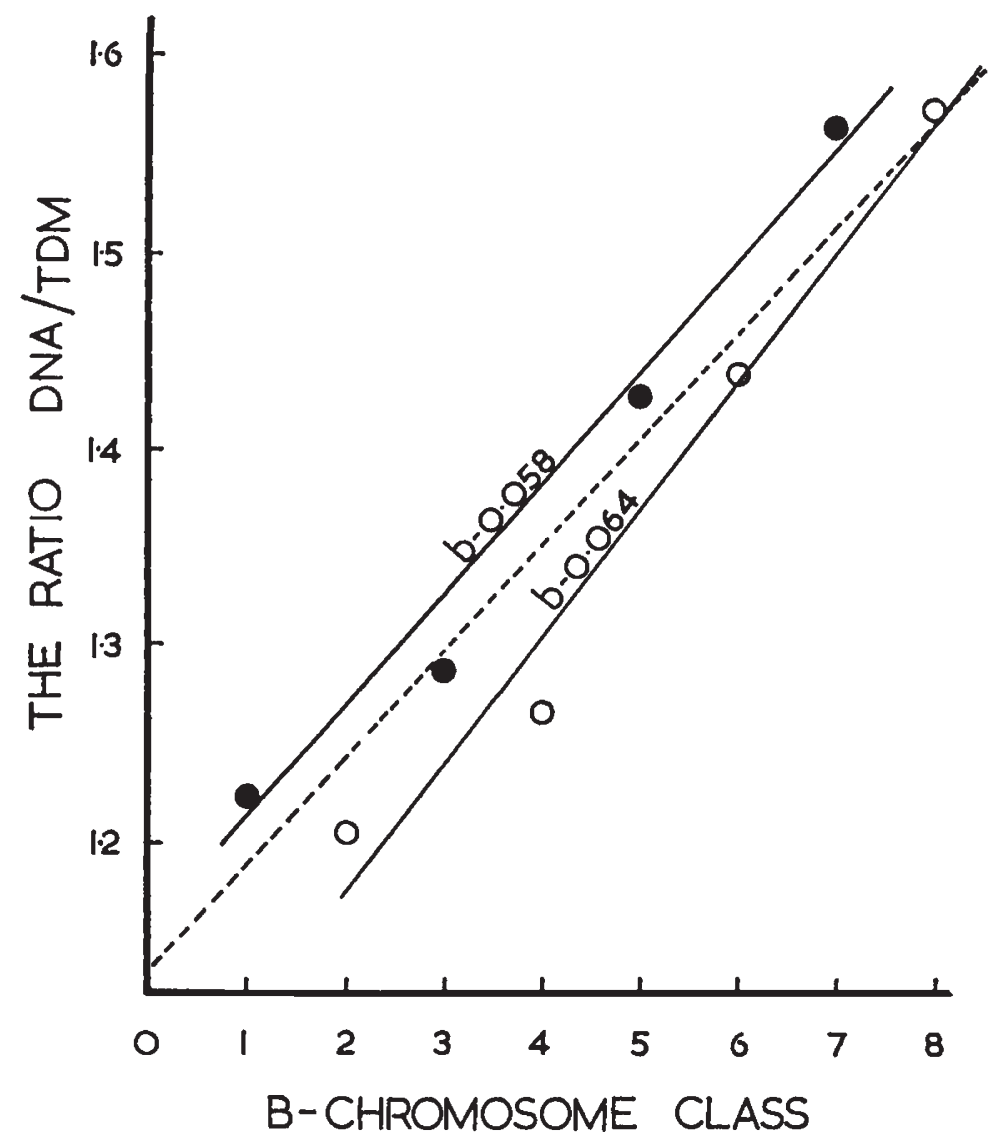

Fig. 3.-The ratio DNA/TDM plotted against: (i) all $B$-chromosome classes, - . - - -; (ii) odd-numbered $B$-classes only, ; (iii) even-numbered $B$-classes only,

number. The results are based on observations in different seasons of many hundreds of plants in three differing rye populations, the commercial Swedish variety Östgöta Gråråg, Wasa II and a primitive Korean strain. The zig-zag relationship between phenotypic expression and alternating odd and even $B$ frequencies is abundantly clear. In fig. 5, which deals with the Korean rye, is also included the average distribution frequencies of $B$ types from seven populations (Müntzing, 1957). It will be seen that the more common $B$ classes, the even numbered, are also those showing greatest vigour as reflected by plant weight, height and tiller number. 
Müntzing also presents extensive data on seed weight, etc. These have not been considered, for a very good reason, viz. that, with open pollination. seeds from the one plant will differ from one another, and from the maternal parent, in $B$ constitution. $B$ effects imposed by the maternal parent will then be confounded by the effects of $B$ 's in the seeds.

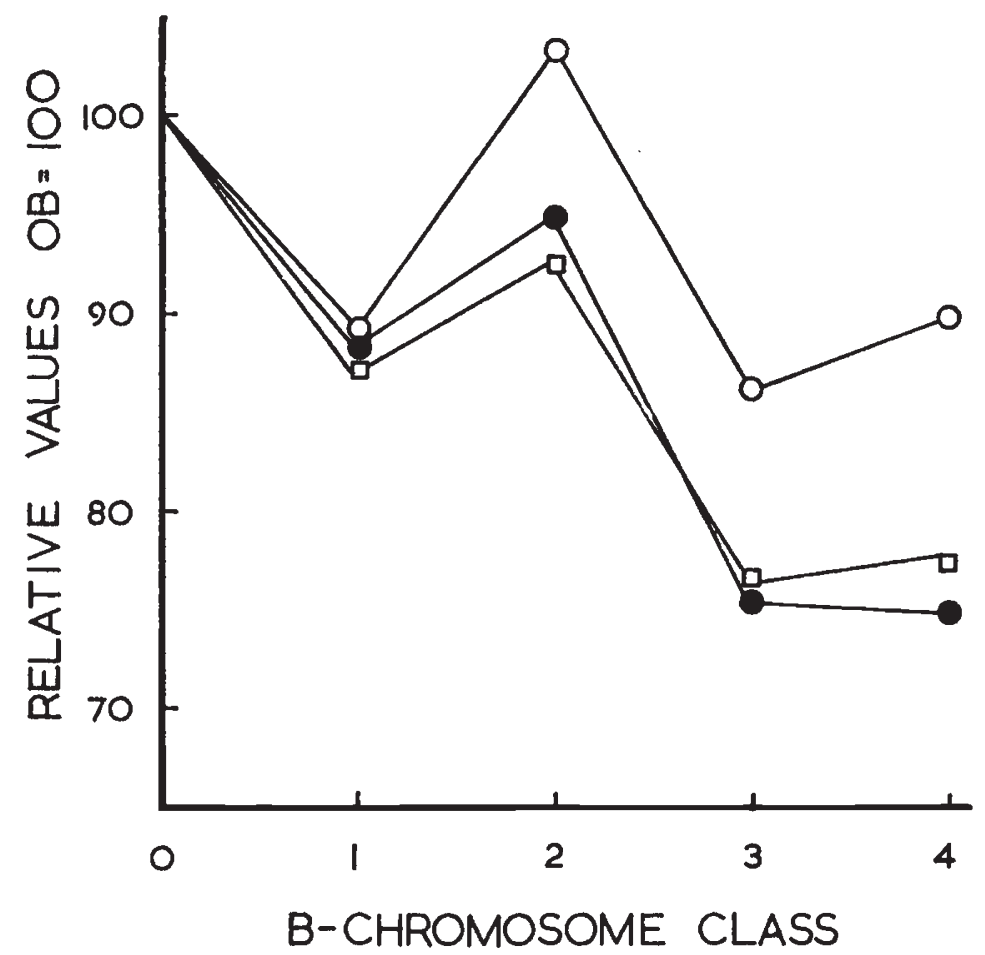

Fig. 4.-Müntzings data (mean values over the three varieties) for: (i) straw weight, $\mathrm{O}-\mathrm{O}$; (ii) tiller number, $\square-\square$; (iii) plant weight, plotted against $B$-chromosome class.

\section{Discussion}

Genetic activity of a simple additive nature will not account for the unusual variation in phenotype that depends on the presence of odd as compared with even numbers of $B$ chromosomes. The only feasible possibility is an interaction of the kind whereby $B$ chromosomes function differently in complete rather than incomplete pairs. Implicit in such a proposal is that their genetic activity would involve and be influenced by a somatic "pairing" or contiguity in interphase nuclei. A situation of almost precisely this kind has recently been established in Drosophila. We need consider only one example (Ashburner, 1967). In a cross between "vg6", which carries a puff at $64 \mathrm{c}$ in polytene nuclei, and "Oregon", which does not, a puff appears in the "Oregon" chromosome at $64 \mathrm{c}$ when there is intimate, homologous pairing at that region. Without such pairing no puff appears in the Oregon chromosome and puffing, it is well known, reflects a localised genetic activity. While there is no direct evidence for a somatic pairing of $B$ 's in rye it is not unreasonable, in the light of the 
Drosophila work, to suggest that their differential activity in odd and even combinations may stem from their altered activity when closely associated in pairs. Indeed, it is difficult to conceive of a satisfactory alternative.

While the causal basis of $B$-controlled variation awaits confirmation, it is worth noting that the differences between odd and even $B$ plants makes

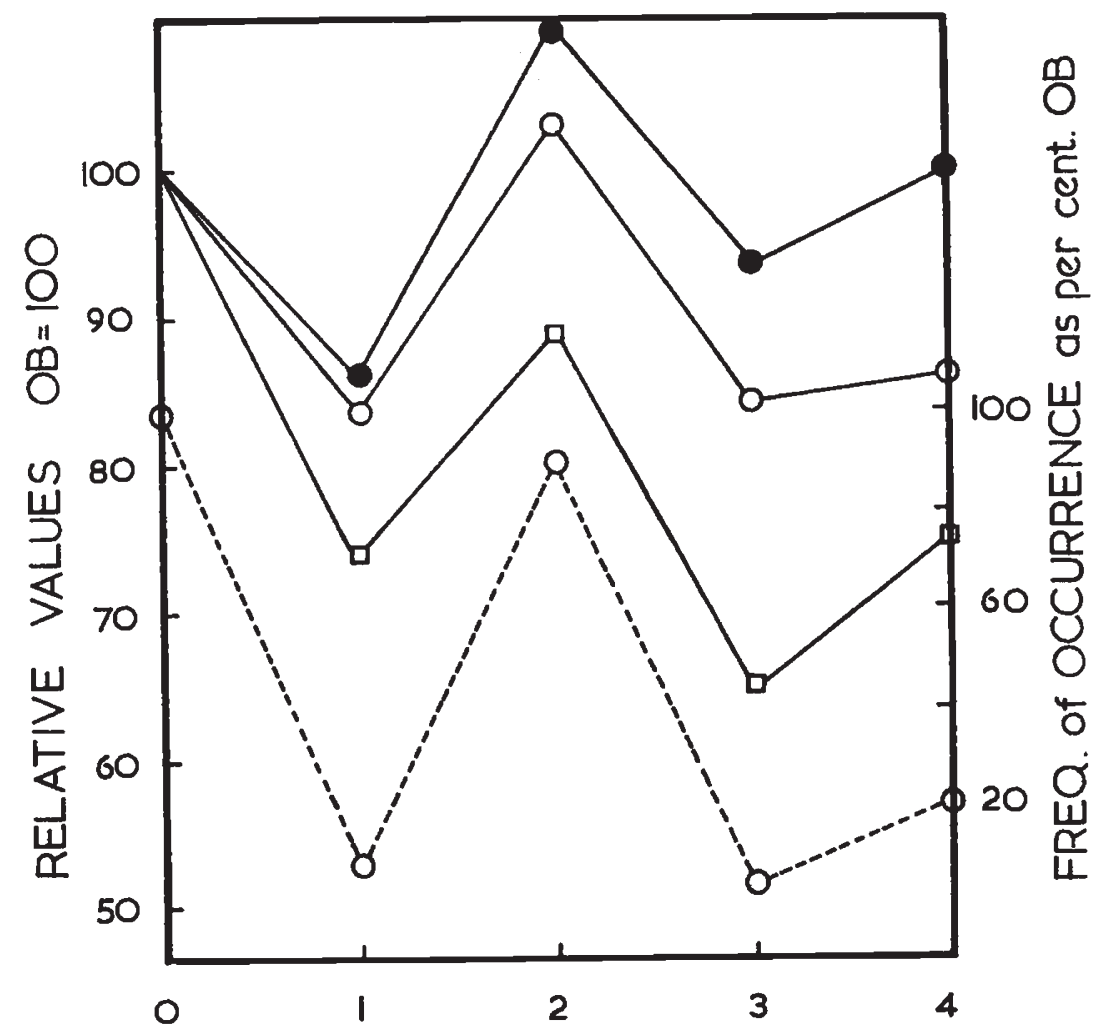

\section{B-CHROMOSOME CLASS}

FIG. 5.-(a) Müntzing's data for three characters of Korean rye: (i) straw weight, ; (ii) plant weight, $\mathrm{C}-\mathrm{O}$; (iii) tiller number, $\square-\square$; plotted against $B$-chromosome class. (b) Müntzing's data for the average frequency of occurrence of different classes of $B$-chromosomes, from seven populations of Korean rye, $\bigcirc-\ldots . . .0$.

considerable "sense" when viewed from the standpoint of $B$ chromosome distribution in rye populations. An excess of even-numbered $B$ plants is the inevitable consequence of a non-disjunction of $B$ 's following both male and female meiosis (Hasegawa, 1934; Müntzing, 1946; Håkansson, 1948). That the more numerous even numbered $B$ plants are disproportionately "vigorous" (see fig. 5) could well be a consequence of a selection promoting an enhanced activity in paired combinations. Alternatively, and to be awkward, one could ascribe to the non-disjunction following meiosis the role of promoting and maintaining the more effective even-numbered $B$ combinations! 


\section{Summary}

1. The influence of $B$ chromosomes upon the rye phenotype differs according to whether the $B$ 's occur in odd as distinct from even numbers.

2. It is suggested that the differential influence of odd and even $B$ frequencies may depend on an altered genetic activity of $B$ 's in contiguous pairs in interphase nuclei.

Acknowledgment.-We thank Professor Müntzing for permission to use his data.

\section{REFERENCES}

ASHBURNER, M. 1967. Gene activity dependent on chromosome synapsis in the polytene chromosomes of Drosophila melanogaster. Nature, 214, 1159-1160.

DARLINGTON, C. D., AND HAQUE, ASHRAFAL. 1966. Organisation of DNA synthesis in rye chromosomes. Chromosomes Today, 1, 102-107.

HÅKANSSON, A. 1948. Behaviour of accessory rye chromosomes in the embryo sac. Hereditas, 34, 35-59.

HASEGAWA, N. 1934. A cytological study on 8-chromosome rye. Cytologia, 6 (1), 68-77.

JONES, R. N., AND REEs, H. 1967. Genotypic control of chromosome behaviour in rye. $\mathrm{XI}$. The influence of $B$ chromosomes on meiosis. Heredity, 22, 333-347.

JONES, R. N., AND REES, H. 1968 . The influence of $B$-chromosomes upon the nuclear phenotype in rye. Chromosoma. (In press.)

KIshiKaWA, H. 1965. Cytogenetic studies of $B$-chromosomes in rye, Secale cereale L., in Japan. Ag. Bull. of Saga University, Oct. 1965, 1-81.

moss, J. P. 1966. The adaptive significance of $B$ chromosomes in rye. Chromosomes Today, $1,15-23$.

MÜNTZING, A. 1946. Cytological studies of extra fragment chromosomes in rye. III. The mechanism of non-disjunction at the pollen mitosis. Hereditas, 32, 97-119.

MÜNTZING, A. 1957. Frequency of accessory chromosomes in rye strains from Iran and Korea. Hereditas, 43, 682-685.

Müntzing, A. 1963. Effects of accessory chromosomes in diploid and tetraploid rye. Hereditas, 49, 361-426. 